\title{
Perceptions of Cancer of Unknown Primary Site - A National Survey of Australian Medical Oncologists
}

Authors: Christos S Karapetis, Lisa Guccione, Martin HN Tattersall, Helen Gooden, Claire M Vajdic, Sylvie Lambert, Monica Robotin, Linda Mileshkin, Penelope Schofield

Date: $4^{\text {th }}$ October 2016

Abstract word Count:

Main document word count:

2999

Authors (in order)

1.Christos S Karapetis

2. Lisa Guccione

3. Martin HN Tattersall

4. Helen Gooden

5. Claire M Vajdic

6. Sylvie Lambert

7. Monica Robotin

8. Linda Mileshkin

9. Penelope Schofield

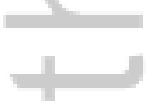

Address for correspondence:
Affiliation Flinders University and Flinders Medical Centre Adelaide, SA, Australia Peter MacCallum Cancer Centre, Parkville, Victoria, Australia Sydney Medical School and Chris $0^{1}$ Brien Lifehouse. Sydney, NSW, Australia Cancer Australia Sydney, NSW, Australia Cancer Epidemiology Research Unit Centre for Big Data Research in Health University of New South Wales Sydney, NSW, Australia Ingram School of Nursing, McGill University St. Mary's Research Centre Montreal, Quebec, Canada School of Medicine, University of Notre Dame, Sydney Faculty of Medicine, School of Public Health, University of Sydney Sydney, NSW, Australia Peter MacCallum Cancer Centre Parkville, VIC, Australia Department of Psychological Sciences School of Health Sciences Swinburne University Melbourne, VIC, Australia A/Prof Chris Karapetis Email: c.karapetis@flinders.edu.au Phone: +61 882048997

Fax: +61882044997

Address: Dept of Medical Oncology Flinders Medical Centre Level 4, Flinders Centre for Innovation in Cancer Bedford Park, SA 5042 Australia

This Ackthewedthement. has not been through the copyediting, typesetting, pagination and proofreading process, which may lead to differences between this version and the Version of Record. Please cite this article
Council NSW. The authors acknowledge the contributions by Jane Barrett, patient and CUP as doi: 10.1111 imj.13373

advocate in the design of the study. 
Abstract:

Introduction: Despite being the $6^{\text {th }}$ cause of cancer death in Australia, cancer of unknown primary (CUP) site remains poorly understood.

Aims: To describe practices relating to the diagnosis, investigation, classification, communication and management of CUP amongst medical oncologists.

Methods: We invited all members of the Medical Oncology Group of Australia to participate in a national, anonymous online survey about CUP. The survey collected data regarding diagnosis acceptance, diagnostic tests, treatment protocols and communication practices around the diagnosis of CUP.

Results: 302 oncologists were invited and 86 (28\%) completed the survey. Eighty respondents (93\%) were directly involved in the assessment of patients with CUP. Eighty-five (99\%) respondents were prepared to make a diagnosis of CUP if, after appropriate diagnostic tests, the primary location could not be ascertained. 83\% would assign a primary site to obtain Pharmaceutical Benefits Schedule (PBS) funding of medical therapy. 62\% did not have a specific treatment protocol designed for CUP. The majority of oncologists used serum tumour markers and CT scans in the initial work-up, whilst $43 \%$ indicated they would use a PET scan in the majority of cases. The majority would arrange mammography in female patients. Thematic analysis of responses to openended questions about how CUP is described identified little consistency in the language being used.

Conclusion: The approach to diagnosis, investigation and management of CUP by medical oncologists in Australia is variable. Many preferred to estimate the primary site and treat accordingly. PBS restrictions may encourage the practice of 'best guessing'.

KEY WORDS: Cancer of Unknown Primary, CUP, Survey, Diagnosis, Communication, Language 


\section{Perceptions of Cancer of Unknown Primary Site - A National Survey of Australian Medical Oncologists}

\section{Introduction}

Failure to determine the site of origin of a cancer, so called cancer of unknown primary site (CUP), has been recognised as a distinct diagnostic entity for over 30 years(1). There are no distinguishing clinical signs or symptoms to denote the primary location of the cancer. Epidemiologic studies have reported that CUP represents between 2 to $5 \%$ of all cancer diagnoses(2) (3). In 2011, approximately 2,800 Australians received a new diagnosis of CUP. This was the $12^{\text {th }}$ most commonly diagnosed cancer in men and $11^{\text {th }}$ in women(4). CUP is typically associated with a poor survival, and in 2012 it was the $6^{\text {th }}$ most common cause of cancer related death (4) .

Recent epidemiological data from the USA and Australia indicate that the incidence of CUP has declined, $(4,5)$ An analysis of the Surveillance, Epidemiology and End Results (SEER) database in the United States revealed a proportional decline in CUP since 2007 to less than 2\% of all cancer diagnoses(6). This decrease may reflect changing clinical diagnostic criteria and reporting practices(7).

Poor acceptance or lack of acknowledgement of a diagnosis of CUP may contribute to an under-reporting of the diagnosis to cancer registries. Whilst in recent years the utilisation of more sensitive radiological procedures, including MRI, fine slice CT scan and positron emission tomography (PET) scanning has increased the detection rate of primary cancers, for the majority of CUP cases the primary cancer remains elusive(8, 9). Doubt regarding the utility and cost effectiveness of these diagnostic procedures for CUP remains, but they may be performed on an individual indication basis $(10,11)$. Immunohistochemical staining of pathology slides has also helped clinicians in ascribing a probable site of origin(12). Most recently the use of gene expression profiling to develop tissue of origin assays has increased the ability to assign the most likely primary 
site(13-18). Despite technological advancements and increased sophistication in the analysis and interpretation of these findings, there usually remains a margin of uncertainty.

Arguably, the diagnosis and management of CUP is one of the most difficult challenges in oncology. Overall, the prognosis for patients with CUP is poor. A recent population-based US registry analysis of adenocarcinoma of unknown primary revealed only a marginal improvement in survival between 1973 and 2008(6). The diagnosis of CUP commonly confuses and frustrates patients, their carers and their doctors. The inability to identify a primary may be seen as a failure of the diagnostic process. Moreover, patients understand that treatment is usually selected according to the site of origin of the malignancy. If a site is not identified, then patients may feel that accurate and optimal treatment selection is not possible.

In many parts of the world, including Australia, access to cancer therapies is regulated. In Australia, access to cancer therapies is funded by government through the public-access Pharmaceutical Benefits Scheme (PBS). The PBS enables subsidised access to relatively expensive cancer treatments for all Australians. Funding approval requires the doctor to gain authority to prescribe particular drugs for specified diagnoses and clinical situations. Specific drugs are funded and made available only for patients with certain cancers.Consequently, oncologists may decide to assign an originating cancer site, so as to enable access to drugs to treat the CUP.

The aim of this study was to assess diagnosis acceptance, diagnostic tests and treatment protocols, as well as communication and documentation practices, amongst a representative sample of Australian medical oncologists.

\section{Methods}

This article is protected by copyright. All rights reserved. 


\section{Sample}

A total of 302 members of the Medical Oncology Group of Australia, who comprise the majority of medical oncologists in Australia, were invited to complete an on-line survey.

\section{Measures}

The survey comprised 14 closed-response questions, and 2 open-ended questions and one categorical question with a comment option. The survey was pilot tested by the CUP steering committee coordinated by the Cancer Council NSW and the wording of items was altered in response to feedback. The survey evaluated oncologists' understanding of CUP and assessed recognition of the diagnosis. The survey also explored the investigations and procedures commonly performed, the treatment protocols utilised, and clinical practices such as communication strategies in the management of CUP. In addition, we asked about the diagnostic assignment, particularly with reference to accessing cancer medication on the PBS, an Australian Commonwealth government funded drug access program. The open-ended questions were designed to explore the language medical oncologists used to describe CUP and how the diagnosis is communicated and discussed. The open ended questions were 'How do you describe or discuss a diagnosis with patients and families? Is CUP a term that you use? Please give a brief description"; and "Cancer of Unknown Primary is a difficult diagnosis to explain. How do you discuss it?" A copy of the questionnaire is provided in the online Supplement

\section{Procedures}

Members of the Medical Oncology Group of Australia were sent an invitation by e-mail, inviting them to complete an online survey. The invitation was initially sent on March 2012 with a reminder email sent in May 2012. The survey was expected to take 10 minutes to complete.

$\underline{\text { Analysis }}$

Descriptive statistics were used to summarise the survey data. A qualitative content analysis of the open-ended questions and of comments provided was also performed. The data was inserted into an Eexcel spreadsheet and two data coders (LG and PS) individually analysed to identify themes and sub-themes. 
The two sets of categorisations were compared and disagreements discussed until agreement was reached.

\section{Results}

A total of eighty-six oncologists completed the survey (response rate 28\%). Eighty of the respondents confirmed their direct involvement in the assessment of patients with CUP.

Participant demographics are presented in Table 1. Seventy-seven of the respondents were based in a metropolitan centre and 70 listed a public hospital or clinic as their principal place of work. The remaining 16 were based in private clinics.

Almost all survey respondents (99\%) stated they were prepared to make a final diagnosis of CUP if after appropriate diagnostic tests the primary site remained uncertain. However, when asked a different question that explored oncologists' preference rather than willingness, $27 \%$ stated that they would prefer to provide a best guess of the primary site of the cancer, rather than diagnose CUP as a specific diagnostic entity, while a further 33\% said that they would sometimes do this

When asked specifically about accessing cancer therapies through the PBS, 83\% $(71 / 86)$ of respondents stated that they document a possible primary location of the cancer and define the cancer as such, to obtain PBS funding of a medical therapy for patients with CUP. Only 15 of the respondents said that they do not do this.

The vast majority (98\%) disagreed "that all CUP cancers are the same entity". Approximately half (51\%) responded 'yes' to the question "Do you have a specific diagnostic process for patients that present with CUP?" and 62\% 
(53/86) stated they did not have a specific treatment protocol designed for patients with CUP at their institution.

The majority of medical oncologists used serum tumour markers and CT scan imaging in the initial diagnostic evaluation of suspected CUP, whilst $43 \%$ indicated they would use a PET scan in at least $50 \%$ of cases. $64 \%$ stated that they would arrange mammography in more than $90 \%$ of cases of female patients with suspected CUP. Medical oncologists performed the following investigations in the majority (> 50\%) of CUP patients; tumour markers (88\%), CT scan (98\%), mammography in female patients (85\%) and gastrointestinal endoscopy (60\%). When medical oncologists were asked about investigations they perform in the vast majority (> 90\%) of CUP patients, only three investigations were included: tumour markers (67\%), CT scan (91\%), mammography in female patients (64\%). The preferred investigations for CUP are depicted in figure 1.

When asked about PET scan use to identify the primary site, $16 \%$ of respondents would arrange a PET scan in $>90 \%$ of CUP cases, $41 \%$ of respondents would arrange a PET scan in $>50 \%$ of CUP cases and the majority either do not utilise PET, or use PET in fewer than $50 \%$ of cases.

\section{Theme 1: Language to describe CUP}

\section{The primary tumour has regressed or is too small}

Clinicians that used the term CUP with their patients also often used the analogy of the primary tumour regressing and hence being undetectable. Several clinicians reported explaining to patients that "Primary (parent) appears to have regressed but metastases (children) have grown", they "explain how in approximately 5\% of cases we never can find the primary as it may be too small to visualise before it sends off metastatic cancer cells to other organs" and "in a percentage of patients we cannot find the primary site as it may have regressed or is not able to be found by conventional diagnostic means". A medical oncologist also reported using this scenario and further elaborating to explain, "there are a 
small number of patients where this happens and it is called 'cancer of unknown primary'”.

\section{Uncertainty}

There is also considerable reference to uncertainty when explaining CUP to patients and family. It is a common subtheme used by medical oncologists both with and without the definitive use of the word CUP. Medical oncologists noted describing CUP as "Cancer that has started in the body and spread but not sure where in the body it started", "occasionally we remain uncertain of where cancer started even after all tests are complete" and also "explain that [it is] uncertain as to where primary malignancy commenced and that sometimes this is the case with cancer".

\section{Poor prognosis}

A minority of medical oncologists reported discussing the diagnosis in terms of its poor prognosis, for example; "whilst we can offer chemotherapy, it is not curable and prognosis is often in the realm of months rather than years"; and "this is a worse prognostic group with a particularly short survival"; "CUP is generally not curable except in occasional circumstances". Another similar recorded response was; "I believe that true CUP is a syndrome with median survival 6 months no matter what you do". Clinicians that did not use the term CUP also described the diagnosis in terms of the associated poor prognosis; "I provide a general description of the behavior of the cancer and the poor prognosis".

\section{'Best guess' diagnosis}

The use of a 'best guess' approach was also reported amongst clinicians who use the term CUP and those who do not. "No I don't use CUP; I usually talk about my best guess primary" and "CUP is a term that I do use, but I try to give information about the most likely primary site based on pathological findings". There were several responses that incorporated a best guess in their description of a likely diagnosis when explaining CUP to their patients, "I divide the body into regions, discuss tissue diagnosis and exclude what we can as a primary"; "I provide a possible origin or most likely site of primary tumour". 


\section{Common cancer diagnosis}

Some responses demonstrated efforts to promote an understanding that this is a recognised common cancer type, I "do tell patients that CUP is a reality and more common than one might think", "it is not unusual to have cancer spread without knowing where it has come from", I "explain that is not uncommon" and I "stress that it is an entity that accounts for up to 5\% of cancer diagnoses".

\section{Theme 2: Treatment approaches}

\section{'Best guess' to guide treatment}

When discussing treatment options to CUP patients, a common subtheme was using the 'best guess' of the primary site to guide treatment choice. Medical oncologists reported that they "explain the uncertainty about diagnosis and best guess the treatment option", "anti-cancer treatment, if appropriate, is based on best guess according to clinical pattern" and the "need to treat with a best guess approach". It was also reported that other clinical information is often used to guide this approach; I "discuss patient presentation, test results and outcomes and best fit chemotherapy for the situation" and "options are based on the location of the disease and likely best fit pattern to determine treatment choice". This approach indicates the use of best clinical judgement in the face of uncertainty. The 'best guess' is based on all available clinical, radiological and histopathological information.

\section{Broad-based therapy and treatments that 'work'}

Medical oncologists also reported explaining the use of treatment effective against a broad spectrum of cancers as opposed to therapies typically used to treat particular cancer types; "There are a small proportion of cancers we are unable to find a primary for and we use therapies which are broad spectrum to give them the best result."; "there are drugs with broader spectrum that may cover a number or possibilities" and "the initial site of the cancer can't be found at this time, so we need to choose drugs that will work across a range of cancers". 
Some medical oncologists were positive about the effectiveness of therapy despite the inability to identify a primary; "we sometimes never find the origin of the cancer and it is usually widespread at diagnosis. It can respond well to treatment". Some were also reassuring patients that there were alternative treatments if the initial treatment was ineffective; "it is still possible to provide treatment and that treatment can be changed to alternative treatment if it is not helping".

\section{Theme 3: Communication challenges}

\section{Clinician skill and time}

Clinician skill, and allocating sufficient time, was highlighted as important in overcoming communication challenges inherent in a CUP diagnosis. Medical oncologists reported the explanation of CUP to be a "discussion [that] is long and involved", "not easily explained in a short comment", and "takes great skill, experience, knowledge, empathy and time".

\section{Patient ability to understand}

A clinician's use of the term CUP was reported to dependent of the patients' level of understanding; I "sometimes use 'CUP' terminology - depending on patient level of understanding". It was also reported that a diagnosis of CUP was "not difficult to explain but difficult for patients and families to understand/accept".

\section{Discussion}

CUP presents a wide diversity in diagnostic and treatment pathways. In the majority of cases, CUP represents a cancer (or a group of cancers) with multiple sites of involvement, displaying relatively aggressive biological behaviour and associated with a poor prognosis, but favourable prognostic subsets are recognised(19-21) (22). Given the complexity around the diagnosis, and the negative connotations associated with terms of uncertainty such as 'unknown', some experts in the field have recommended changing the diagnostic term to 
"Cancer of Complex Origin"(23). Our survey indicates that the language around CUP and medical oncologists' approach to the initial diagnosis, investigation and management of CUP in Australia is variable. To our knowledge this is the first attempt to understand how oncologists manage CUP clinically and the challenges that they face. We are not aware of any similar published data that we can compare our results to.

Many clinicians preferred to use of best clinical judgement to guess the primary site of the cancer and treat accordingly. In Australia, PBS restrictions to drug access appear to encourage the practice of 'best guessing' the primary site of cancer origin and assigning a diagnosis when requesting subsidised drug access via the PBS. The PBS restrictions otherwise prejudice CUP patients accessing potentially effective treatments. Acceptance of the CUP diagnosis was not universal amongst Australian oncologists. This may have implications regarding the accuracy of site-specific cancer registry data in Australia.

Variability was observed in the selection of the preferred initial investigations. CT scans, serum tumour markers, GI endoscopy and mammography (for female patients) are investigations that oncologists said that they performed in the majority of cases. PET scanning is used in less than $50 \%$ of cases. A high degree of variability in the selection of investigations for the evaluation of CUP has previously been reported(1, 24). In recent years, however, attempts to standardise the management of patients with CUP have been published based in the form guidelines produced by the NCCN (National Comprehensive Cancer Network) and the European Society of Medical Oncology $(25,26)$. These guidelines are based on evidence and consensus, but several areas remain contentious. The utility of PET in this setting, for example, remains debatable. Some authors have reported a high rate (>50\%) of primary lesion detection through the application of FDG-PET/CT scans $(27,28)$. Others have reported a sensitivity of less than $50 \%(9,29)$. A recent study observed a high sensitivity, but low specificity in detecting the primary through PET in patients presenting with CUP in cervical nodes, and it is in this group that the guidelines recommend use of PET/CT (30). 
Most oncologists surveyed stated that their institutions did not have CUP specific treatment protocols. This may be attributable to the absence of a globally accepted regimen. The approach to the treatment of CUP is evolving with the expectation that molecular analysis of the tumour will help identify genetic alterations for which there are specific targeted therapies. Indeed, a recent study reported a high rate of detectable genetic alterations in CUP specimens, the majority of which were found to be amenable to a molecular targeted treatment strategy(31). Our survey did not specifically explore the utilisation of molecular testing in the assessment of CUP.

\section{Limitations}

A response rate of $28 \%$ is low and we acknowledge that there is likely to be bias in the responses obtained. Of the respondents within this sample, $94 \%$ of medical oncologists were involved in the assessment of patients during the diagnosis of CUP; therefore data collected in this survey is biased towards oncologists that treat CUP. However, we believe that this is the only data available that assess diagnosis acceptance, diagnostic tests selected and treatment protocols used by medical oncologists. The assessment of the communication and documentation practices in relation to the CUP diagnosis also provides valuable insights into clinical practice and this has never been previously investigated or reported.

\section{Conclusion}

The survey highlighted that CUP is a diagnosis that is accepted by oncologists but the majority would be prepared to provide a 'best guess' of the primary site in order to access drugs on the PBS and provide affordable treatment to patients. The management of patients with CUP is variable, as is the clinical presentation. The use of existing clinical practice guidelines may help Australian clinicians manage this complex malignancy.

This article is protected by copyright. All rights reserved. 
Acknowledgement:

The survey was supported through the CUP steering committee coordinated by the Cancer Council NSW. The authors acknowledge the contributions by Jane Barrett, patient and CUP advocate in the design of the study

Table 1. Demographics of participating medical oncologists.

\begin{tabular}{|c|c|}
\hline$=\sqrt{2}$ & $\begin{array}{l}\text { Oncologists } \\
(\mathrm{N}=86)\end{array}$ \\
\hline \multicolumn{2}{|l|}{ Age (years) } \\
\hline - Less than 40 & $30(35 \%)$ \\
\hline - $40-49$ & $25(29 \%)$ \\
\hline - $\quad 50-59$ & $24(28 \%)$ \\
\hline$\bullet \quad>60$ & $7(8 \%)$ \\
\hline \multicolumn{2}{|l|}{ Principal work location } \\
\hline - Public Hospital/Public Clinic - Capital City & $65(75 \%)$ \\
\hline - Private Hospital/Private Clinic - Capital City & $12(14 \%)$ \\
\hline - Public Hospital/Clinic - Regional & $5(6 \%)$ \\
\hline - Private Hospital/Private Clinic - Regional & $4(5 \%)$ \\
\hline \multicolumn{2}{|l|}{ State or Territory } \\
\hline - Australian Capital Territory & $5(6 \%)$ \\
\hline - New South Wales & $21(24 \%)$ \\
\hline - Victoria & $30(35 \%)$ \\
\hline - Queensland & $10(12 \%)$ \\
\hline - South Australia & $11(13 \%)$ \\
\hline - Western Australia & $3(3 \%)$ \\
\hline - Tasmania & $6(7 \%)$ \\
\hline - Northern Territory & $0(0 \%)$ \\
\hline
\end{tabular}

This article is protected by copyright. All rights reserved. 
Figure 1: Responses to Question: What investigations do you typically use to diagnose CUP?

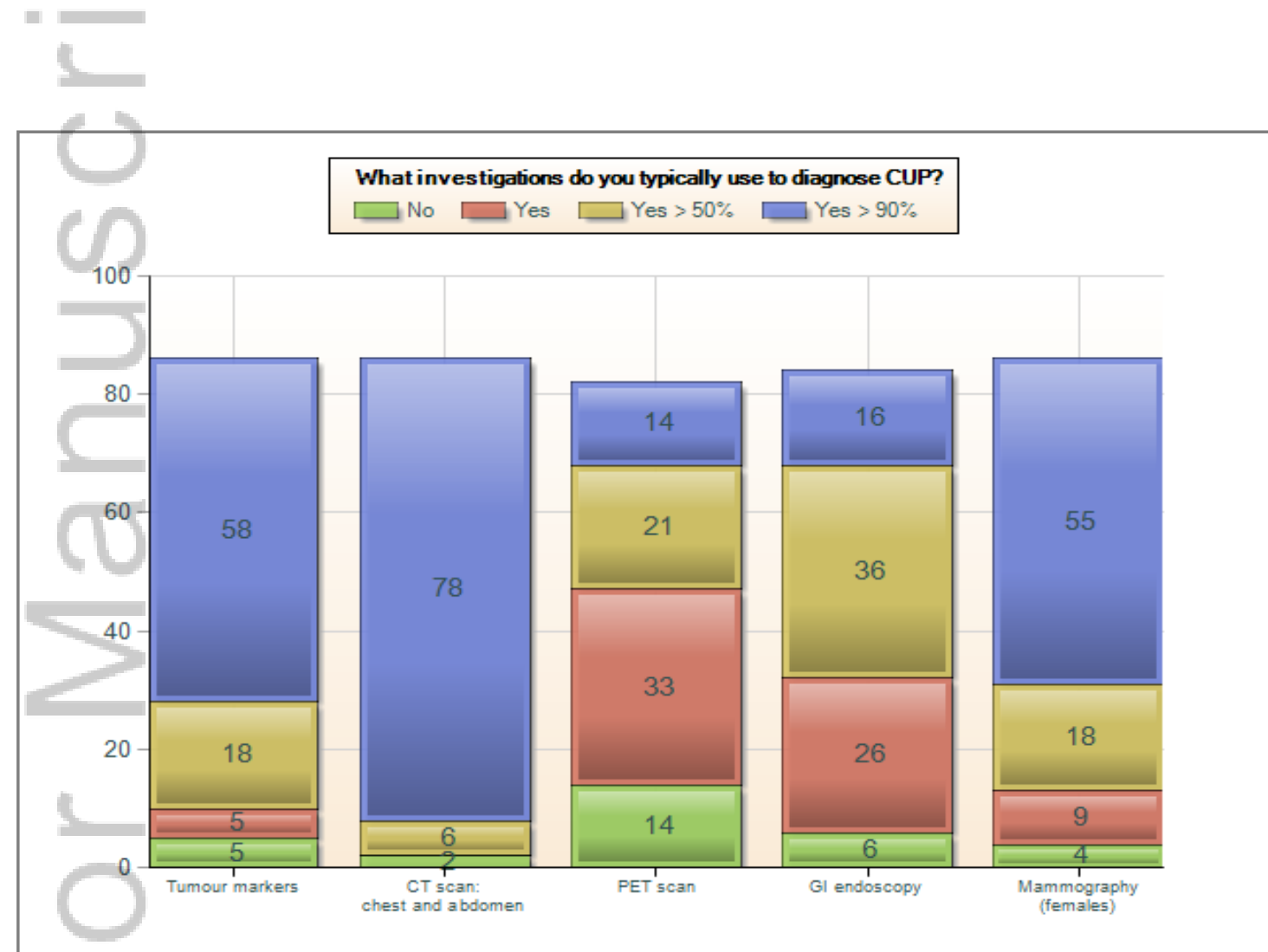

( $\mathrm{y}$ axis $=$ number of respondents)

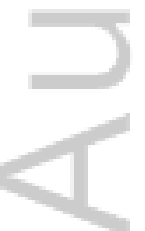

This article is protected by copyright. All rights reserved. 
1. Stewart JF, Tattersall MHN, Woods RL, Fox RM. Unknown primary adenocarcinoma: Incidence of over-investigation and natural history. British Medical Journal 1979; 1:1530-3.

2. Muir C. Cancer of unknown primary site. Cancer.1995;75(1 Suppl):353-6.

3. Tracey EA GP, Roder D, Currow D, Jelfs P, Bishop J. Unknown primary cancer in New South Wales. Sydney: Cancer Institute NSW Monograph; 2008.

4. Australian Institute of Health and Welfare 2014. Cancer in Australia: an overview 2014. Cancer series No 90. Cat. no. CAN 88. Canberra: AIHW

5. Mnatsakanyan E, Tung WC, Caine B, Smith-Gagen J. Cancer of unknown primary: Time trends in incidence, United States. Cancer Causes \& Control 2014;25(6):747-57.

6. Urban D, Rao A, Bressel M, Lawrence YR, Mileshkin L. Cancer of unknown primary: a population-based analysis of temporal change and socioeconomic disparities. British Journal of Cancer 2013;109(5):1318-24.

7. Vajdic CM, Wyld L, Symons JN. The challenge of attributing causality in cancer of unknown primary. International Journal of Cancer 2013;133(5):1266-7. 8. Moller AK, Loft A, Berthelsen AK, al. e. 18F-FDG PET/CT as a diagnostic tool in patients with extracervical carcinoma of unknown primary site: a literature review. The Oncologist 2011;16:445-51.

9. Park JS, Yim JJ, Kang WJ, Chung JK, Yoo CG, Kim YW, et al. Detection of primary sites in unknown primary tumors using FDG-PET or FDG-PET/CT. BMC Research Notes 2011;4:56.

10. Levine MN, Drummond MF, Labelle RJ. Cost-effectiveness in the diagnosis and treatment of carcinoma of unknown primary origin. Canadian Medical Association Journal 1985;133(10):977-87.

11. Varadhachary GR, Raber MN. Carcinoma of unknown primary site. The New England Journal of Medicine 2014;371(21):2040.

12. Hashimoto K, Sasajima Y, Ando M, Yonemori K, Hirakawa A, Furuta K, et al. Immunohistochemical profile for unknown primary adenocarcinoma. PloS One 2012; 7(1):e31181.

13. Varadhachary GR, Talantov D, Raber MN, Meng C, Hess KR, Jatkoe T, et al. Molecular profiling of carcinoma of unknown primary and correlation with clinical evaluation. Journal of Clinical Oncology 2008;26(27):4442-8.

14. Horlings HM, van Laar RK, Kerst JM, Helgason HH, Wesseling J, van der Hoeven JJ, et al. Gene expression profiling to identify the histogenetic origin of metastatic adenocarcinomas of unknown primary. Journal of Clinical Oncology 2008;26(27):4435-41.

15. Bridgewater J, van Laar R, Floore A, Van TVL. Gene expression profiling may improve diagnosis in patients with carcinoma of unknown primary. British Journal of Cancer 2008;98(8):1425-30.

16. Hainsworth JD, Rubin MS, Spigel DR, Boccia RV, Raby S, Quinn R, et al. Molecular gene expression profiling to predict the tissue of origin and direct sitespecific therapy in patients with carcinoma of unknown primary site: a prospective trial of the Sarah Cannon research institute. Journal of Clinical Oncology 2013;31(2):217-23. 
17. Gross-Goupil M, Massard C, Lesimple T, Merrouche Y, Blot E, Loriot Y, et al. Identifying the primary site using gene expression profiling in patients with carcinoma of an unknown primary (CUP): a feasibility study from the GEFCAPI. Onkologie. 2012;35(1-2):54-5.

18. Chiang WM, Kapadia M, Laver NV, Nystrom JS. Cancer of unknown primary: from immunohistochemistry to gene expression profiling. Journal of Clinical Oncology 2012;30(29):e300-2.

19. Greco FA, Vaughn WK, Hainsworth JD. Advanced poorly differentiated carcinoma of unknown primary site: recognition of a treatable syndrome. Annals of Internal Medicine. 1986;104(4):547-53.

20. Altman E, Cadman E. An analysis of 1539 patients with cancer of unknown primary site. Cancer 1986;57(1):120-4.

21. Kirsten F, Chi CH, Leary JA, Ng AB, Hedley DW, Tattersall MH. Metastatic adeno or undifferentiated carcinoma from an unknown primary site--natural history and guidelines for identification of treatable subsets. The Quarterly Journal of Medicine 1987;62(238):143-61.

22. Fizazi K. Treatment of patients with specific subsets of carcinoma of an unknown primary site. Annals of Oncology 2006;17 Suppl 10:x177-80.

23. Daud AI. Removing the unknown from the carcinoma of unknown primary. Journal of Clinical Oncology 2013;31(2):174-5.

24. Shaw PH, Adams R, Jordan C, Crosby TD. A clinical review of the investigation and management of carcinoma of unknown primary in a single cancer network. Clin Oncol (R Coll Radiol). 2007;19(1):87-95.

25. National Comprehensive Cancer Network. Occult Primary Guidelines Version 2.2014 (Cancer of Unknown Primary [CUP] 2016 Available from: https://www.nccn.org/professionals/physician gls/pdf/occult.pdf.

26. Fizazi K, Greco FA, Pavlidis N, Daugaard G, Oien K, Pentheroudakis G, et al. Cancers of unknown primary site: ESMO Clinical Practice Guidelines for diagnosis, treatment and follow-up. Annals of Oncology 2015;26 Suppl 5:v133-8.

27. Fencl P, Belohlavek O, Skopalova M, Jaruskova M, Kantorova I, Simonova K. Prognostic and diagnostic accuracy of [18F]FDG-PET/CT in 190 patients with carcinoma of unknown primary. European Journal of Nuclear Medicine and Molecular Imaging 2007;34(11):1783-92.

28. Kaya AO, Coskun U, Unlu M, Akdemir UO, Ozdemir NY, Zengin N, et al. Whole body 18F-FDG PET/CT imaging in the detection of primary tumours in patients with a metastatic carcinoma of unknown origin. Asian Pacific Journal of Cancer Prevention 2008;9(4):683-6.

29. Kole AC, Nieweg OE, Pruim J, Hoekstra HJ, Koops HS, Roodenburg JL, et al. Detection of unknown occult primary tumors using positron emission tomography. Cancer 1998;82(6):1160-6.

30. Zhu L, Wang N. 18F-fluorodeoxyglucose positron emission tomographycomputed tomography as a diagnostic tool in patients with cervical nodal metastases of unknown primary site: a meta-analysis. Surgical Oncology 2013;22(3):190-4.

31. Ross JS, Wang K, Gay L, Otto GA, White E, Iwanik K, et al. Comprehensive Genomic Profiling of Carcinoma of Unknown Primary Site New Routes to Targeted Therapies. JAMA Oncology 2015; 


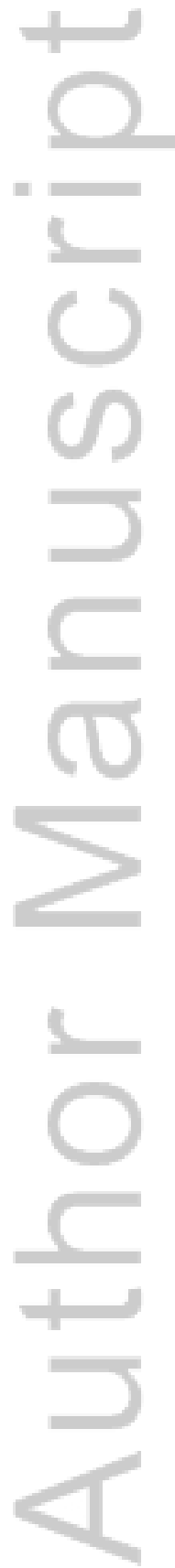

This article is protected by copyright. All rights reserved. 
Abstract:

Introduction: Despite being the $\underline{6}^{\text {th }}$ cause of cancer death in Australia, cancer of unknown primary (CUP) site remains poorly understood.

Aims: To describe practices relating to the diagnosis, investigation, classification, communication and management of CUP amongst medical oncologists.

Methods: We invited all members of the Medical Oncology Group of Australia to participate in a national, anonymous online survey about CUP. The survey collected data regarding diagnosis acceptance, diagnostic tests, treatment protocols and communication practices around the diagnosis of CUP.

Results: 302 oncologists were invited and 86 (28\%) completed the survey. Eighty respondents (93\%) were directly involved in the assessment of patients with CUP. Eighty-five (99\%) respondents were prepared to make a diagnosis of CUP if, after appropriate diagnostic tests, the primary location could not be ascertained. 83\% would assign a primary site to obtain Pharmaceutical Benefits Schedule (PBS) funding of medical therapy. 62\% did not have a specific treatment protocol designed for CUP. The majority of oncologists used serum tumour markers and CT scans in the initial work-up, whilst 43\% indicated they would use a PET scan in the majority of cases. The majority would arrange mammography in female patients. Thematic analysis of responses to openended questions about how CUP is described identified little consistency in the language being used.

Conclusion: The approach to diagnosis, investigation and management of CUP by medical oncologists in Australia is variable. Many preferred to estimate the primary site and treat accordingly. PBS restrictions may encourage the practice of 'best guessing'.

This article is protected by copyright. All rights reserved. 


\section{University Library}

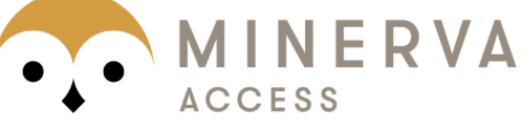

A gateway to Melbourne's research publications

Minerva Access is the Institutional Repository of The University of Melbourne

Author/s:

Karapetis, CS;Guccione, L;Tattersall, MHN;Gooden, H;Vajdic, CM;Lambert, S;Robotin, M;Mileshkin, L;Schofield, P

Title:

Perceptions of cancer of unknown primary site: a national survey of Australian medical oncologists

Date:

2017-04-01

Citation:

Karapetis, C. S., Guccione, L., Tattersall, M. H. N., Gooden, H., Vajdic, C. M., Lambert, S., Robotin, M., Mileshkin, L. \& Schofield, P. (2017). Perceptions of cancer of unknown primary site: a national survey of Australian medical oncologists. INTERNAL MEDICINE JOURNAL, 47 (4), pp.408-414. https://doi.org/10.1111/imj.13373.

Persistent Link:

http://hdl.handle.net/11343/292727 